Scientific Journal of Hamadan Nursing \& Midwifery Faculty - ISSN 2008-2819

\title{
The Study of Symptom-Management's Self-Efficacy in Breast Cancer Survivors Undergoing Chemotherapy
}

\author{
Fatemeh Moghaddam Tabrizi ${ }^{1}$, Saeedeh Alizadeh ${ }^{2 *}$, Samira Barjasteh ${ }^{3}$
}

1. Associate Professor, Department of Nursing and Midwifery, Reproductive Health Research Center, Urmia University of Medical Sciences, Urmia, Iran

2. Midwifery Consultation Masters'Degree Student, Student Research Center, Urmia University of Medical Sciences, Urmia, Iran

3. PhD Students in Reproductive Health, Nursing and Midwifery School, Tehran University of Medical Sciences, Tehran, Iran

\begin{tabular}{lc}
\hline \multicolumn{1}{c}{ Article Info } \\
\hline & \\
Received: & $2017 / 02 / 13$ \\
Accepted: & $2017 / 05 / 7$ \\
Published Online & $2017 / 07 / 17$
\end{tabular}

DOI: $10.30699 /$ sjhnmf.26.5.2

Original Article

Use your device to scan and read the article online

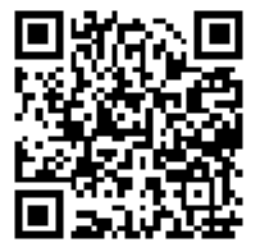

\section{Abstract}

Introduction: Self-efficacy emphasizes on skills and abilities to carry out successful worthy performance. The aim of this study was to evaluate the symptom-management's self-efficacy associated with chemotherapy and its related factors in women referred to academic centers of Urmia in 2016.

Methods: This study was a descriptive-analytical cross-sectional study conducted on 150 patients with breast cancer referred to academic centers of Urmia in 2016 and sampling method was convenience. The collecting data instrument was the symptom-management's self-efficacy scale-breast cancer (SMSES-BC) questionnaire with three subscales including acquiring problem-solving, managing chemotherapy-related symptoms and managing emotional and interpersonal disorders. The data was analyzed using SPSS20.

Results: Most of the patients were in the age range of 41-49. The Lowest scores were in the area of emotional and interpersonal disorders and the highest scores were in the a $\mathrm{r}$ ea of managing chemotherapy-related symptoms. The mean score of s e lf-effi c acy chemotherapy-related symptoms was $167.96 \pm 25.40$. There was a signi $\mathrm{f}$ icant relationship between age $(P=0.024)$, economic status $(P=0.006)$, education, marital status, and life status $(P=0.001)$ and self-efficacy chemotherapy-related symptoms.

Conclusion: Interventional efforts to promote the function of symptom management self-efficacy, especially managing emotional and interpersonal disturbance in these patients are important and necessary.

Keywords: Breast Cancer, Self-Efficacy, Symptom Management

Copyright $($ C 2018, Sci J Hamadan Nurs Midwifery Fac. This is an open-access article distributed under the terms of the Creative Commons Attribution-noncommercial 4.0 International License which permits copy and redistribute the material just in noncommercial usages, provided the original work is properly cited.

How to Cite This Article:

Moghaddam Tabrizi F, Alizadeh S, Barjasteh S. The Study of Symptom-Management's Self-Efficacy in Breast Cancer Survivors Undergoing Chemotherapy. Sci J Hamadan Nurs Midwifery Fac. 2018; 25 (5): $163-171$ 


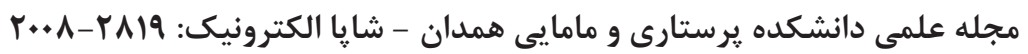

مقالأ يزوهشى همدان

\section{بررسى خودكار آمدى مديريت علايم مرتبط با شيمى درمانى در زنان مبتلا به سرطان يستان}

\section{فاطمه مقدم تبريزى'، سعيده عليز اده ז"، سميرا برجسته}

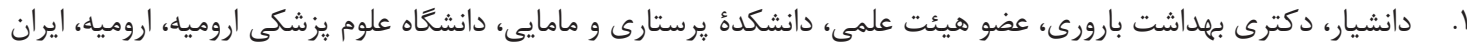

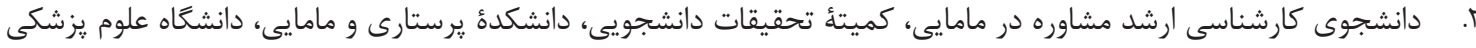

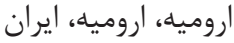

r. دانشجوى دكترى تخصصى بهداشت بارورى، دانشكدة يرستارى و مامايى، دانشعاه علوم يزشكى تهران، تهران، ايران

\begin{tabular}{|c|c|}
\hline جكيده & اطلاعات مقاله \\
\hline 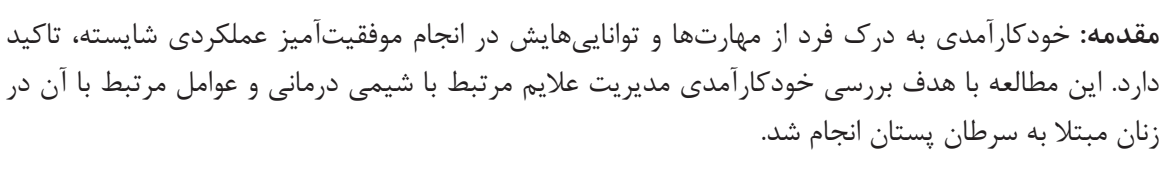 & تاريخ وصول: \\
\hline 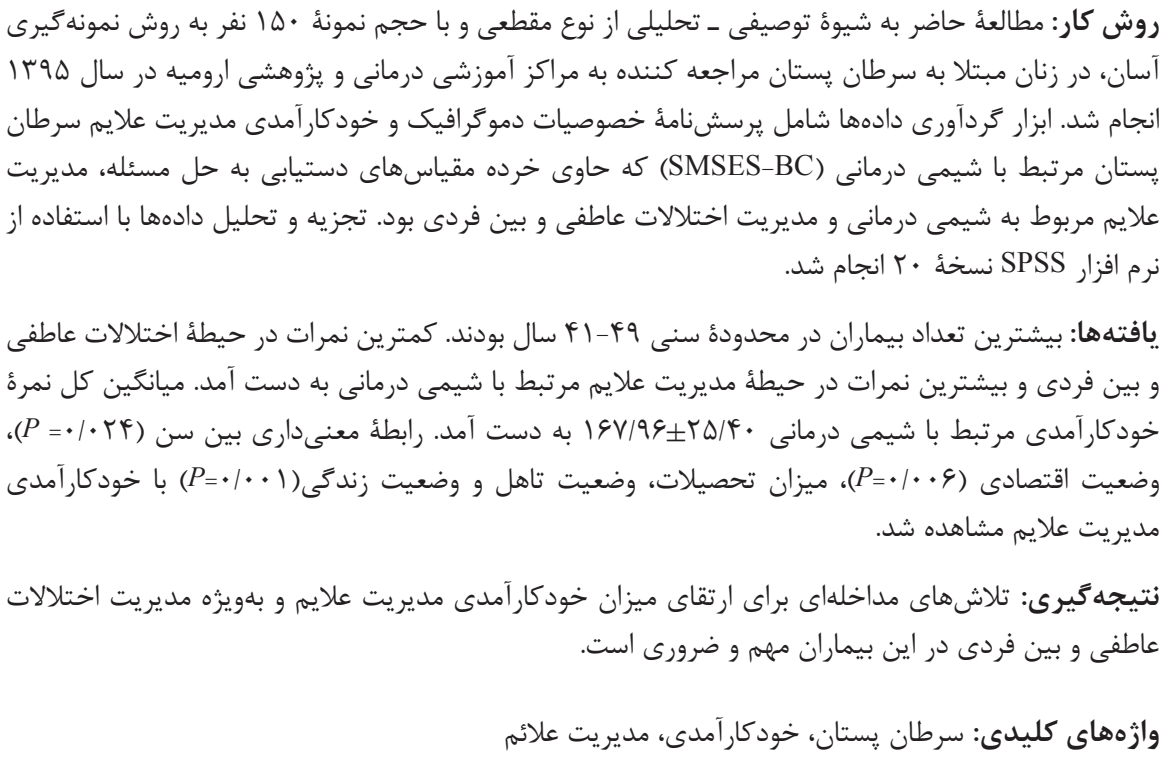 & 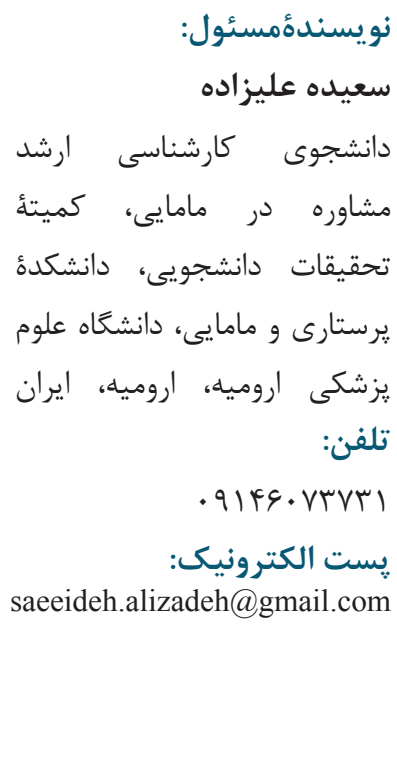 \\
\hline
\end{tabular}

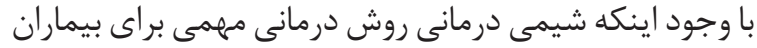

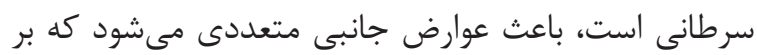

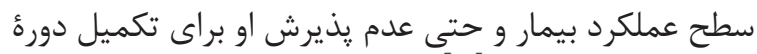

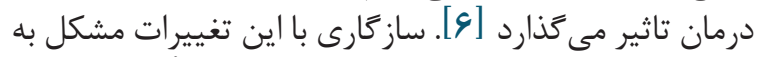

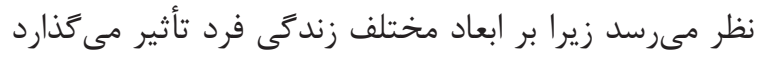

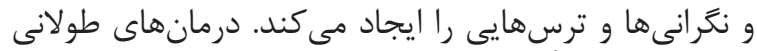

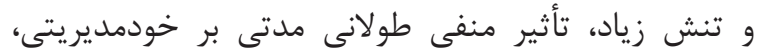

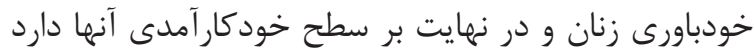

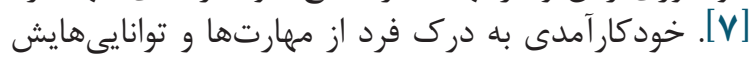

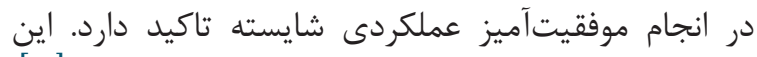

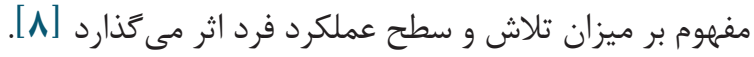
تحقيقات نشان مىدهد زنان مبتلا به سرطان يستان كه به توانايى هاى خود اطمينان دارند، فعالانه در برنان بنانهان بـان

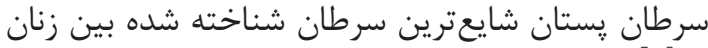

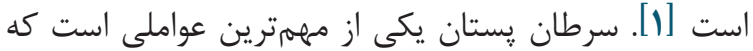

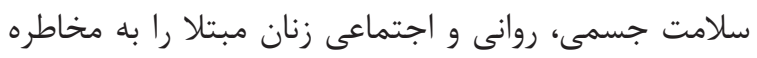

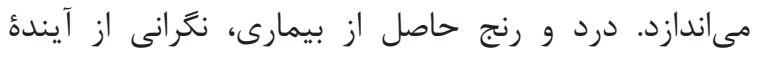

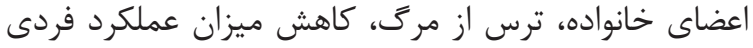

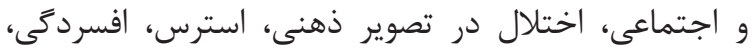

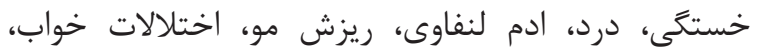

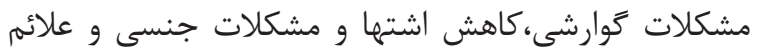

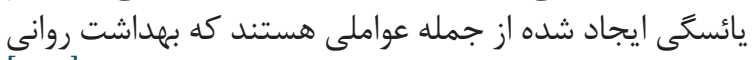

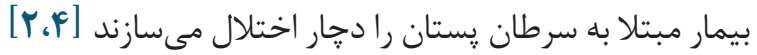

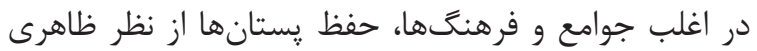

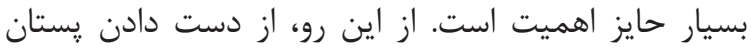
بيشتر به معناى از دست دادن هويت زنانه تعبير مىشود [هان]. 


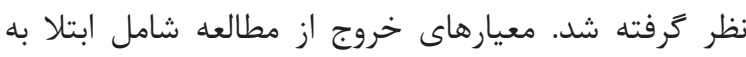

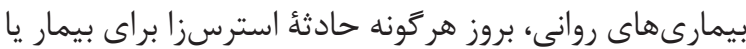

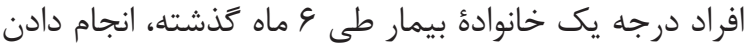

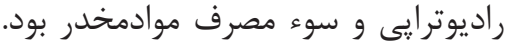

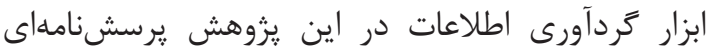

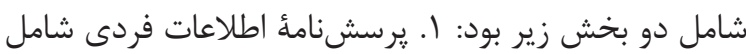

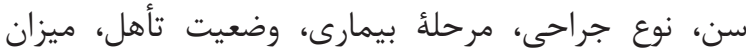

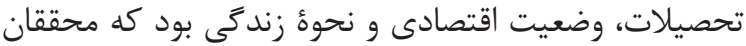

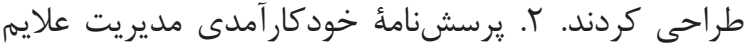

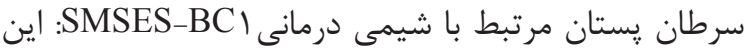

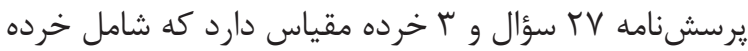

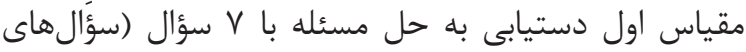

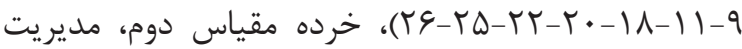
علايم مربوط به شيمى درمانى با هاسؤال (سؤال هاى

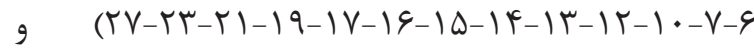

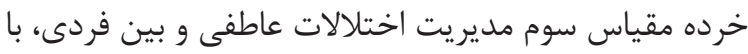

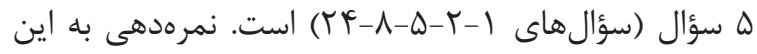

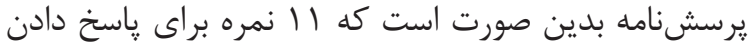

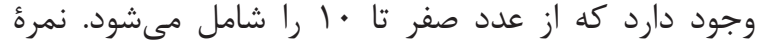

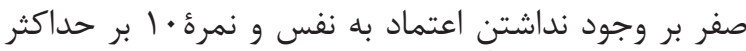

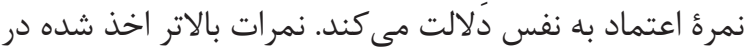

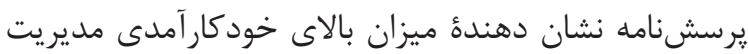

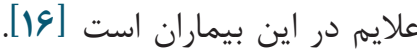

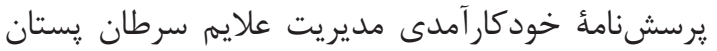

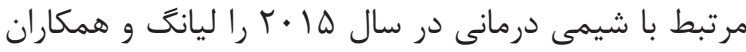

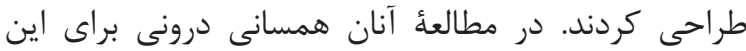

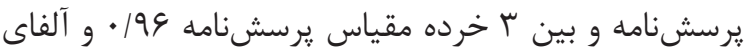

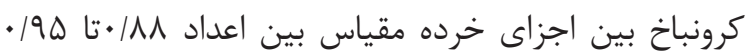

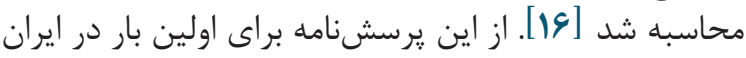

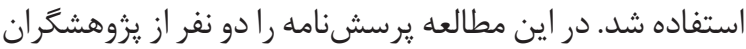

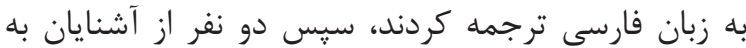

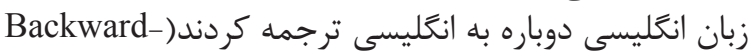

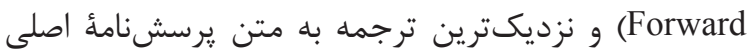

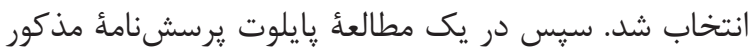

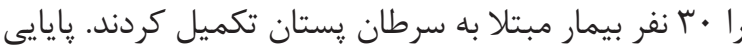

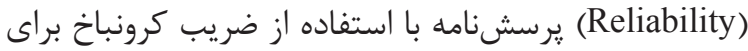

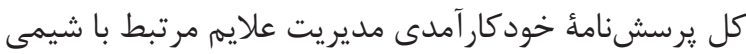

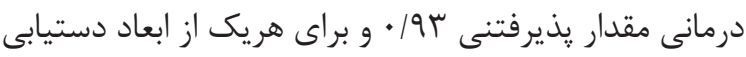

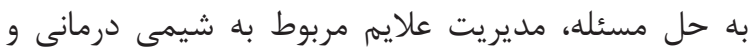

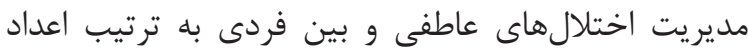

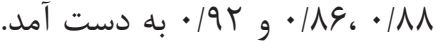

طبق نظر كميتهأ تحقيقات دانشجويى دانشكدة يرستارى

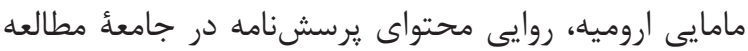

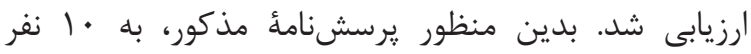

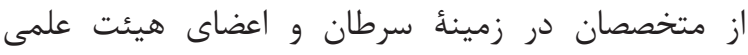

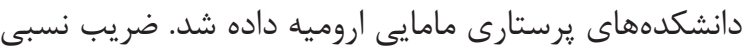

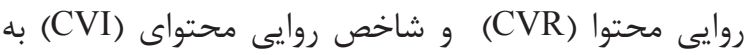
1. Symptom-Management Self-Efficacy Scale for Breast Cancer Related to Chemotherapy
بهداشتى و درمانى شان شركت مى كنند و اين مشار كت سبب

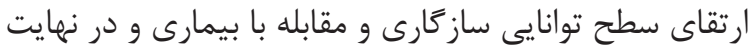

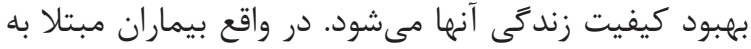

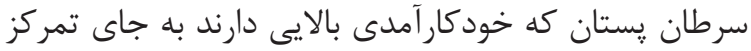

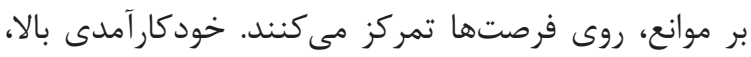

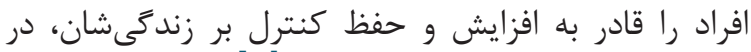

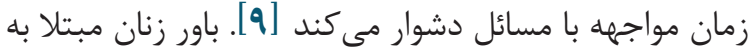

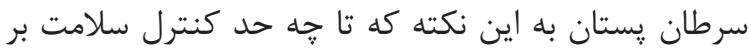

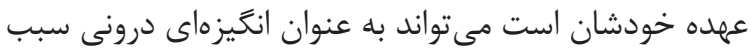

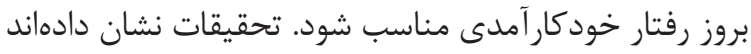

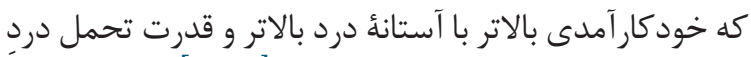

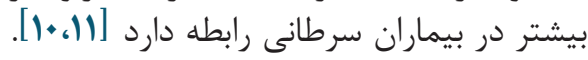

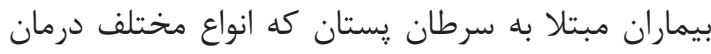

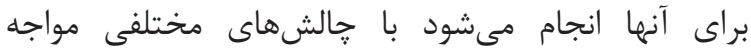

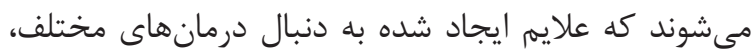

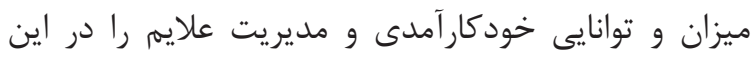

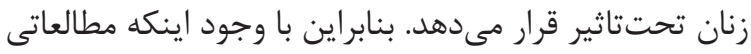

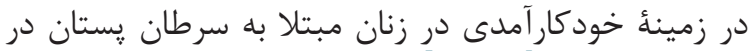

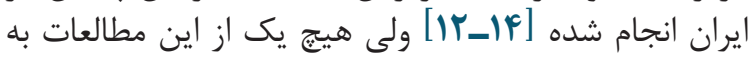

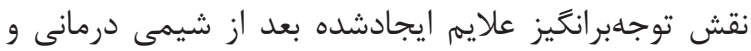

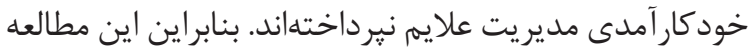

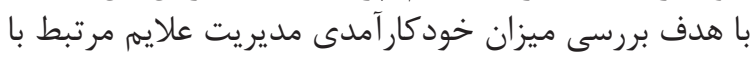

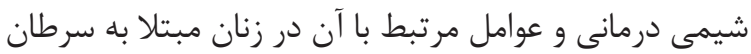

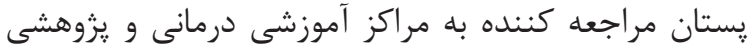

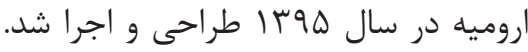

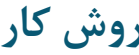

اين يزوهش مطالعهاى توصيفى ـ تحليلى از نوع مقطعى برى بردي

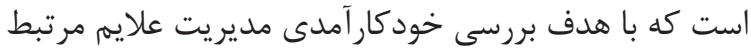

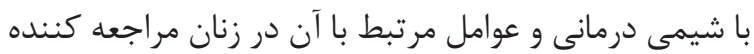

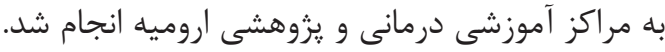

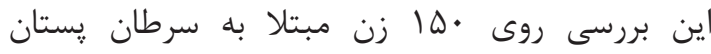

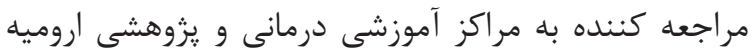
Convenience or Accidental) با روش نمونه (sampling Shelby

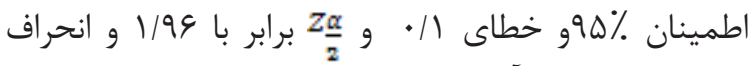

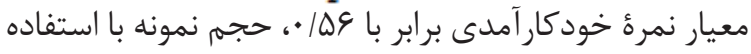

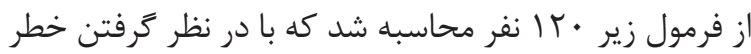

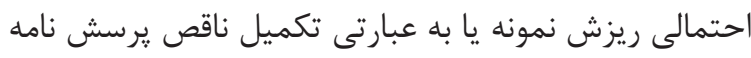

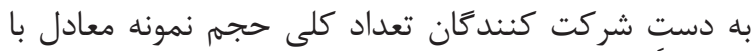

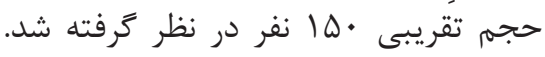

$$
\mathrm{n}=\frac{z_{\frac{\alpha}{2}}^{2} s^{2}}{d^{2}}=120
$$

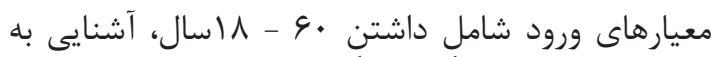

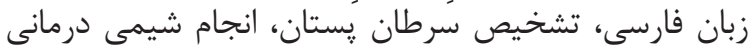

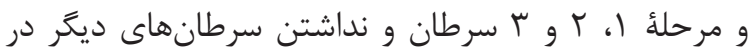




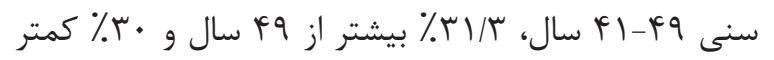

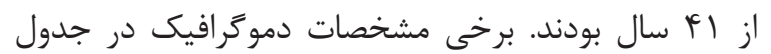
شماره أ أذكر شده است.

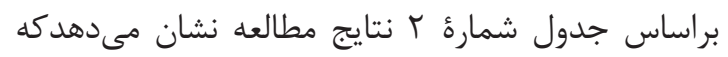

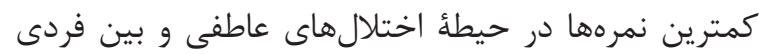
با ميانگَين و انحراف معيار

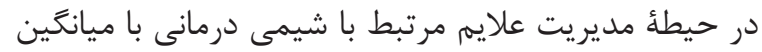

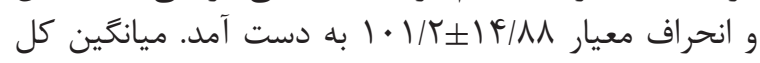

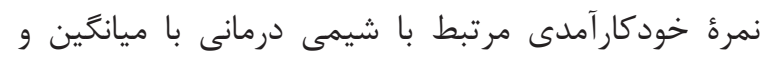

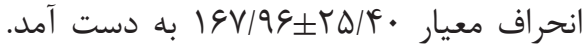

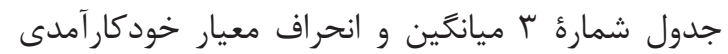

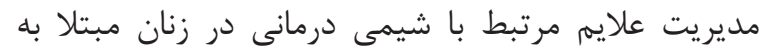

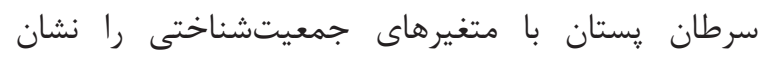

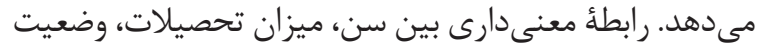

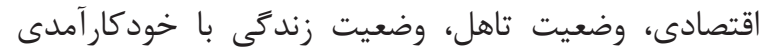

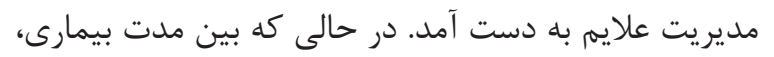

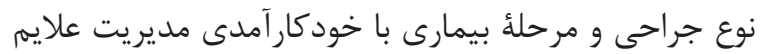

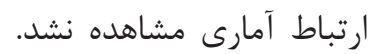

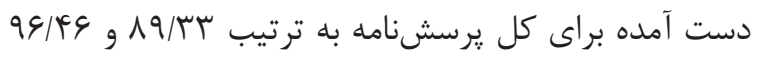
بود كه ميزانى يذيرفتنى است.

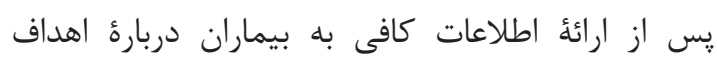

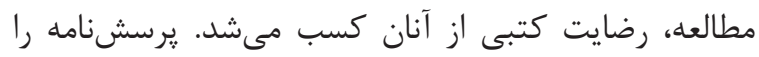

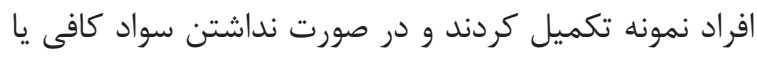

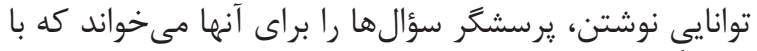

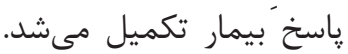

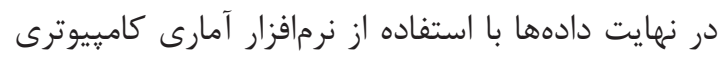

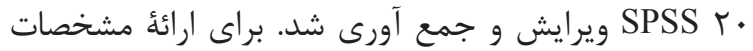

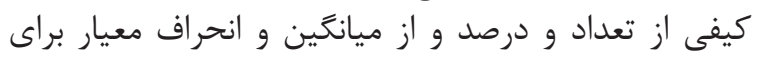
مشخصات كمى استفاده شد. در اين مطالعه از آز آزمون آماري

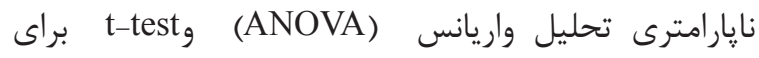

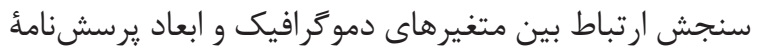

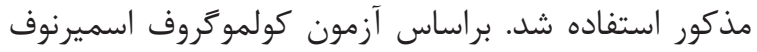

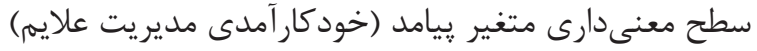

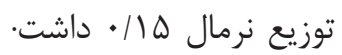

يافتهها

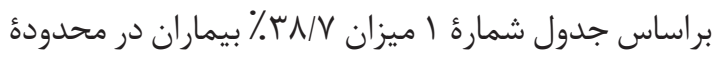

جدول ا.خصوصيات جمعيتشناختى مبتلايان به سرطان يستان

\begin{tabular}{|c|c|c|c|}
\hline 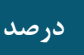 & تعداد & & مشخصات دموكرافيك \\
\hline & & & سن \\
\hline$r \cdot$ & $i \Delta$ & $>+1$ & \\
\hline rA/V & $\Delta \Lambda$ & $41-4 q$ & \\
\hline \multirow[t]{2}{*}{ r } & iv & $4 q<$ & \\
\hline & & & ميزان تحصيلات \\
\hline ir & 11 & بى سواد & \\
\hline$F V / r$ & vi & زير دييلم & \\
\hline$r \& / V$ & f. & دييلم & \\
\hline \multirow[t]{2}{*}{ if } & rl & دانشخاهى & \\
\hline & & & وضعيت اقتصادى \\
\hline$r \& / V$ & f. & دخل كمتر از خرج & \\
\hline$q V / \pi$ & 1.1 & دخل برابر با خرج & \\
\hline \multirow[t]{2}{*}{9} & 9 & دخل بيشتر از خرج & \\
\hline & & & وضعيت تاهل \\
\hline$r / r$ & $\Delta$ & 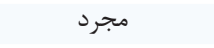 & \\
\hline$\Lambda \varepsilon / V$ & $\pi$ & متاهل & \\
\hline 9 & 9 & مطلقه & \\
\hline \multirow[t]{2}{*}{9} & 4 & بيوه & \\
\hline & & & وضعيت زندگى \\
\hline $19 / \Gamma$ & Irf & همسر و فرزند & \\
\hline$\wedge$ & it & يدر و مادر & \\
\hline \multirow[t]{2}{*}{$T / V$} & f & تنها & \\
\hline & & & مدت بيمارى \\
\hline rt & is & $1-r^{k}$ & \\
\hline FT/V & sq & $\Delta-9$ & \\
\hline \multirow[t]{2}{*}{$r \Delta / r$} & ץ^ & $\vee-\Lambda$ & \\
\hline & & & نوع جراحى \\
\hline$V V / r$ & 119 & ماستكتومى كامل & \\
\hline \multirow[t]{2}{*}{$T r / V$} & rF & ماستكتومى نسبى & \\
\hline & & & مرحله بيمارى \\
\hline$r \cdot / V$ & r & مرحله 1 & \\
\hline$F V / r$ & vi & مرحله r & \\
\hline rt & \&A & مرحله r & \\
\hline
\end{tabular}


سعيده عليزاده و همكاران.

جدول شماره r. يراكندگى، ميانگَين و انحراف معيار هر يك از ابعاد خودكار آمدى مديريت علايم مرتبط با شيمى درمانى در زنان مبتلا به سرطان يستان

\begin{tabular}{|c|c|c|c|c|c|}
\hline انحراف معيار & 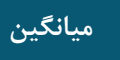 & حداكثر نمره & حداقل نمره & 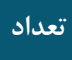 & متغير \\
\hline $9 / 4 q$ & $F T / \Delta F$ & 99 & 10 & 10 & دستيابى به حل مسئله \\
\hline $\mid F / \Lambda \Lambda$ & $1 \cdot 1 / r$ & 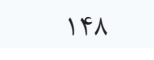 & \&4 & 10 & مديريت علائم مر تبط با شيمى درمانى \\
\hline$g / F V$ & $r \cdot 199$ & is & ir & 10 & مديريت اختلالات عاطفى و بين فردى \\
\hline$r \Delta / f^{\circ}$. & $19 V / 99$ & tre & Ar & 10 & نمرءكل خود كار آمدى مدير يت علايم مرتبط با شيمى درمانى \\
\hline
\end{tabular}

جدول شماره س. ارتباط خودكار آمدى مديريت علائم مرتبط با شيمى درمانى در زنان مبتلا به سرطان يستان با متغيرهاى جمعيتشناختى

\begin{tabular}{|c|c|c|c|c|}
\hline سطح معنى دارى & $\mathbf{F}$ & ميانكين土|نحراف معيار & & متغير \\
\hline \multirow[t]{5}{*}{$\cdot / \cdot r Y^{*}$} & $r / \Lambda$. & $|V \cdot N| \pm T V / A \mid$ & $>+1$ & \multirow{4}{*}{ سن } \\
\hline & & $|V r / \Delta| \pm r \mid / F q$ & $p 1-p q$ & \\
\hline & & $|\Delta 9 / V T \pm r \varepsilon| \cdot G$ & $p q<$ & \\
\hline & & $\mid \varsigma V / Q ९ \pm r \Delta / F$ & Total & \\
\hline & & $\mid F q / V T \pm 19 / \Delta r$ & بي سواد & \multirow{5}{*}{ ميزان تحصيلات } \\
\hline \multirow[t]{5}{*}{$\cdot|\cdot \cdot|^{*}$} & $\Delta / \Delta$ & $\mid \varepsilon V / A Y \pm r \cdot / V \Delta$ & زير دييلم & \\
\hline & & $\mid 99 / 4 T \pm K r / \cdot 9$ & ديبلم & \\
\hline & & $|\wedge| / r r \pm r \mid / 99$ & دانشكاهى & \\
\hline & & $\mid \varepsilon V / 9 \varepsilon \pm r \Delta / 4$. & Total & \\
\hline & & $\mid \Delta q / 1 \Delta \pm r F / F$ & دخل كمتر از خرج & \multirow{4}{*}{ وضعيت اقتصادى } \\
\hline \multirow[t]{4}{*}{$\cdot 1 \cdot .9^{*}$} & $\Delta / r q$ & $199 / \Lambda r \pm r r / \Delta$. & دخل برابر با خرج & \\
\hline & & $\mid \Lambda G / T Y \pm Y \Lambda / r Y$ & دخل بيشتر از خرج & \\
\hline & & 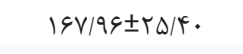 & Total & \\
\hline & & $|r V / r \cdot \pm f| / \Delta \mid$ & 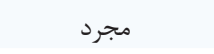 & \multirow{5}{*}{ وضعيت تأهل } \\
\hline \multirow[t]{5}{*}{$\cdot \mid \cdot \cdot 1^{*}$} & $9 / \pi$. & $\mid V \backslash / I \varepsilon \pm T r / V V$ & متاهل & \\
\hline & & $\mid \Delta F / \Delta \Delta \pm r r$ & مطلقه & \\
\hline & & IFF/rTETY/AV & بيوه & \\
\hline & & $\mid \varsigma V / 99 \pm r \Delta / F$ & Total & \\
\hline & & $|V \cdot| \Lambda V \pm r r / G \Lambda$ & همسر و فرزند & \multirow{4}{*}{ وضعيت زندگى } \\
\hline \multirow[t]{4}{*}{$\cdot|\cdot \cdot|^{*}$} & $9 / \% 1$ & $|f Q / \varphi q \pm r \Delta /| Q \Delta$ & يدر و مادر & \\
\hline & & IrV/DITr/Fr & تنهيا & \\
\hline & & $\mid \varepsilon V / ৭ ६ \pm r \Delta / \uparrow$. & Total & \\
\hline & & $\mid 9 q / r T \pm T V / \cdot F$ & $1-r^{2}$ & \multirow{4}{*}{ مدت بيمارى } \\
\hline \multirow[t]{4}{*}{$\cdot / V r$} & $\cdot / r$. & $19 N / 9 \Delta \pm r r / \Lambda$ & $\Delta-9$ & \\
\hline & & $|\varepsilon \Delta / r| \pm r \varepsilon / r \Delta$ & $\vee-\Lambda$ & \\
\hline & & $\mid \varsigma V / 9 \varsigma \pm r \Delta / \uparrow$. & Total & \\
\hline & & $\mid \& 9 / 9 V \pm r \Delta / 9 V$ & ماستكتومى كامل & \multirow{3}{*}{ نوع جراحى } \\
\hline \multirow[t]{3}{*}{.$/ 1 T$} & $\cdot / \mathrm{V} \wedge$ & $|Y| / r \Delta \pm Y r / \Delta \mid$ & ماستكتومى نسبى & \\
\hline & & $\mid \varsigma V / Q ९ \pm r \Delta / \uparrow$ & Total & \\
\hline & & $19 D / G Y \pm T V / T V$ & مرحله | & \multirow{4}{*}{ مرحلة بيمارى } \\
\hline \multirow[t]{3}{*}{.$/ 94$} & $4 / 11$ & $|V T / T Y \pm Y|$ & مرحله r & \\
\hline & & $\mid q r / \cdot r \pm r q / r V$ & مرحله r & \\
\hline & & $19 V / Q 9 \pm \Gamma \Delta / \uparrow$ & Total & \\
\hline
\end{tabular}

* Statistically significant $(P<0.05)$. 
آنها نشان داد، افرادى كه بيمارى آنها در سنين هأ سال و و

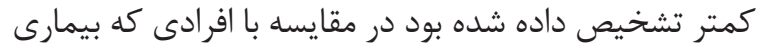

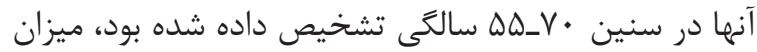

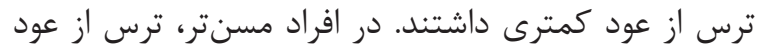

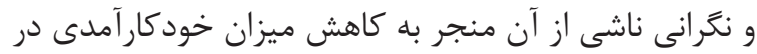

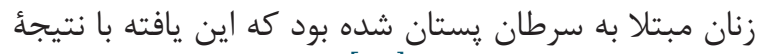

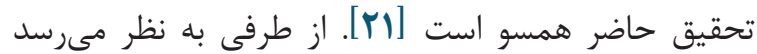

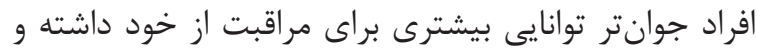

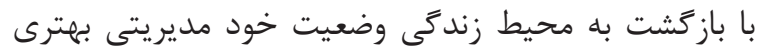
داشته باشند. باز

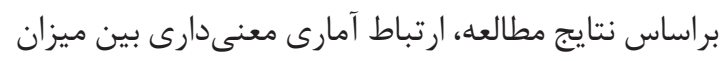

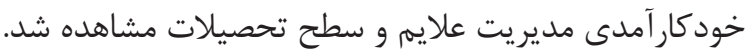

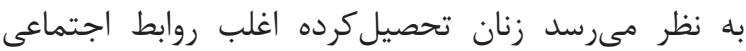

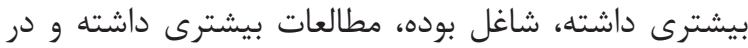

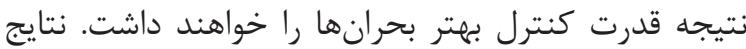

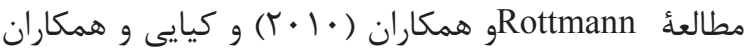

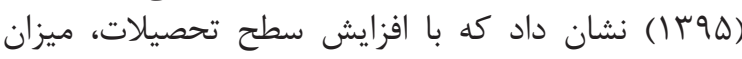

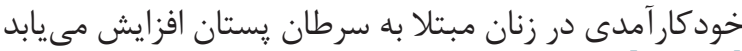

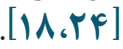

در اين مطالعه ارتباط معنى دارى بين وضعيت اقتصادى

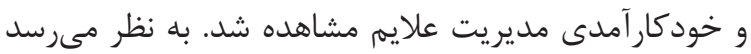

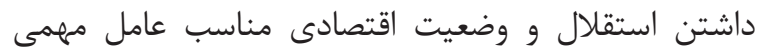

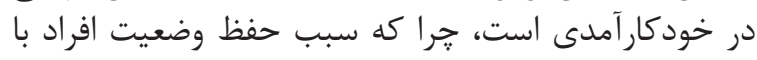

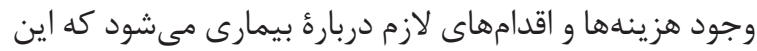

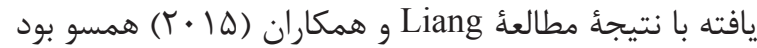

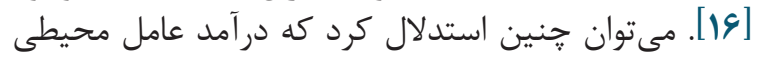

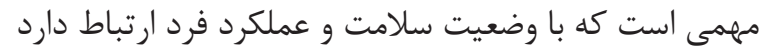

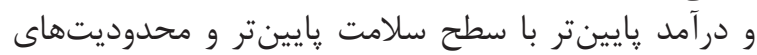

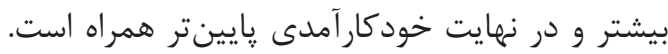

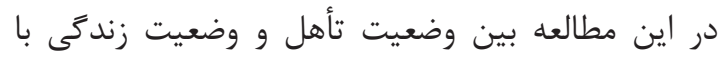
خودكارآمدى مديريت علايم ارئ ارتباطى بسيارى مشاهده شئ شد.

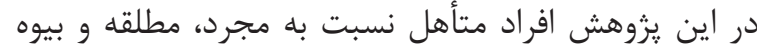

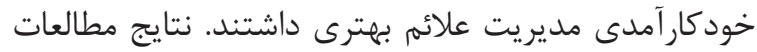

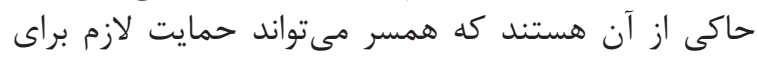

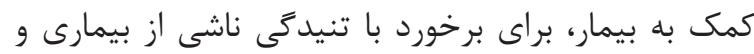

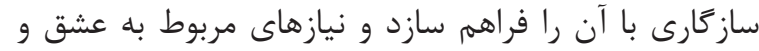

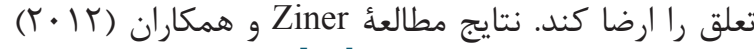
نيز همسو با مطالعه حاضر بود [rإن]

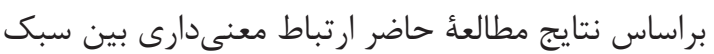

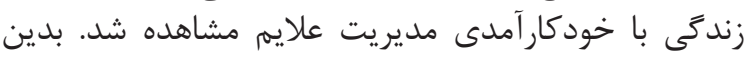

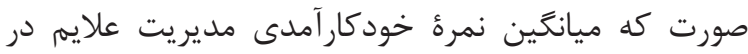

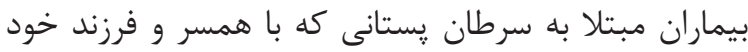

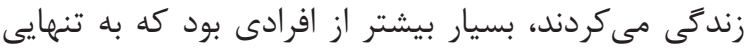

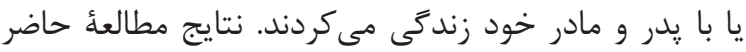

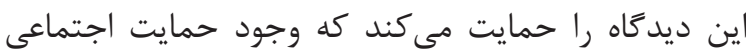

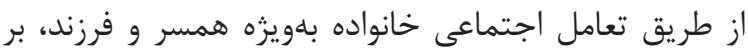

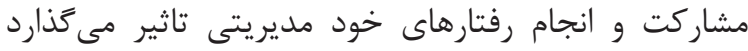
و حمايت اجتماعى كافى، اعمال و افكار فرد راني راني براى انجام

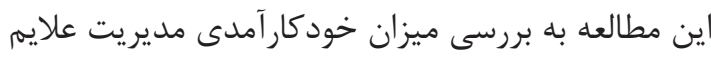

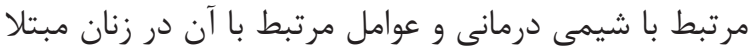

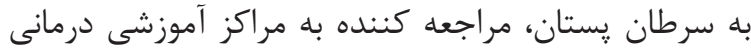

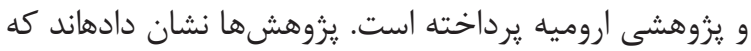

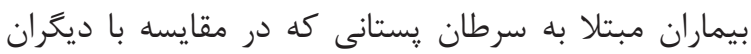

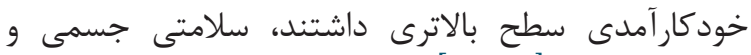

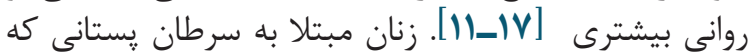

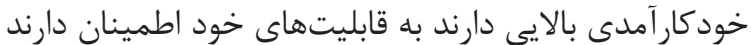

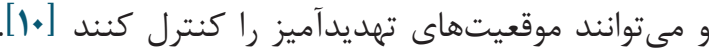

نتايج مطالعهُ حاضر نشان داد تعداد بيشترى از بيماران

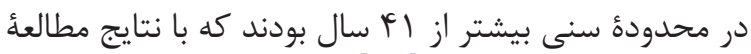

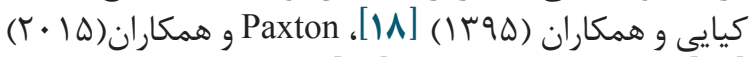
[19] و و Tabrizi

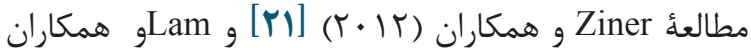

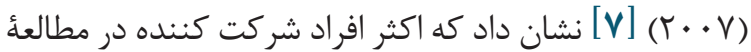

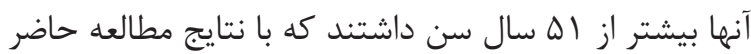

$$
\text { ناهمسو است. }
$$

نتايج مطالعه حاضر نشان داد كه كمترين نمرهٔ ميانكَين

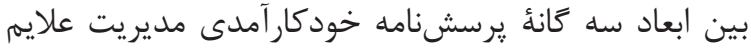

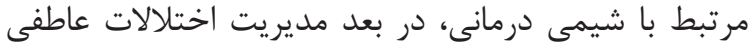

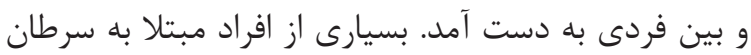

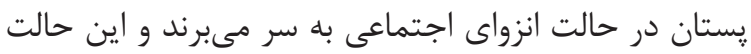

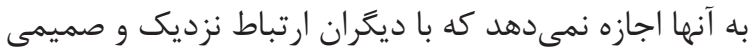

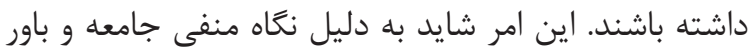

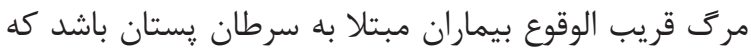

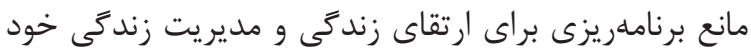

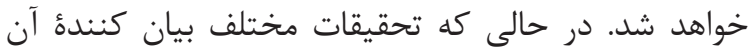

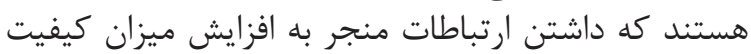

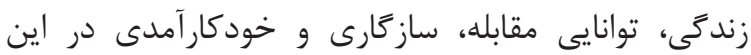

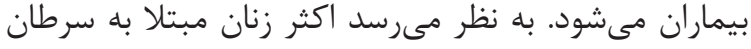

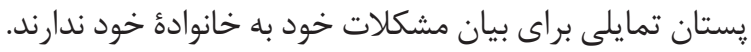

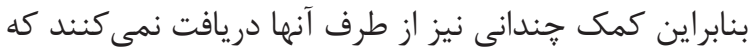

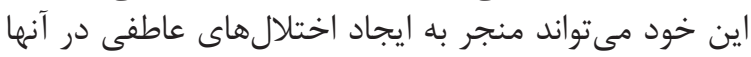

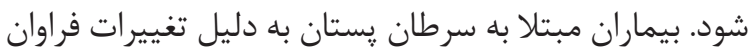

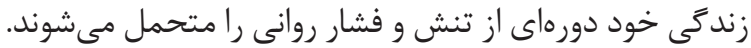

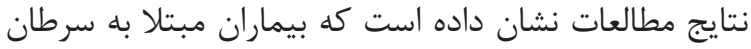

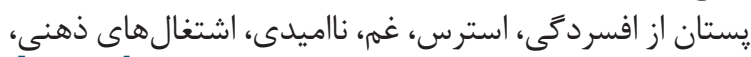

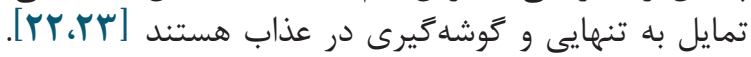

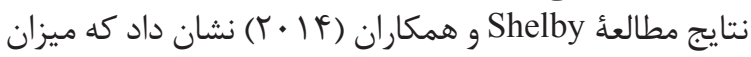

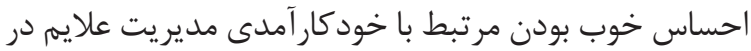

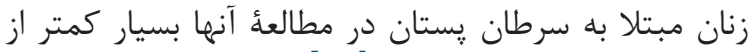

ساير از حيطهها به دست آمد [1/]. نتايج مطالعئ حاضر نشان داد كه بين سن و خود آرار آمدى

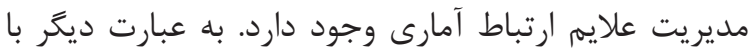

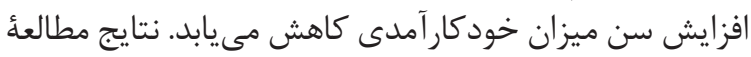
و و همكاران (Tiner سن ميزان ترس از عود سرطان افزايش مى ايابد. نتايج مطالعأ 
خودمديريتى در بيمارستانها و مراكز بهداشتى درمانى از طريق دست اندكاران طرح ريزى و اجران شودان

\section{محدوديت مهاى يثوهش}

اين يزوهش با محدوديتهايى روبرو بود كه ذكر آنها براى

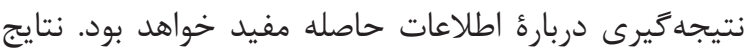

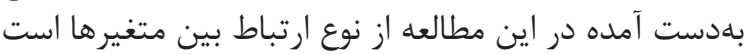

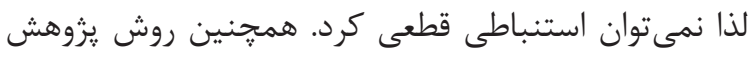

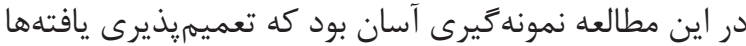

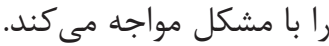

\section{سياسگَزارى}

اين مقاله حاصل طرح تحقيقاتى مصوب كميتهٔ تحقيقات

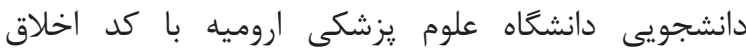
تجات تحقيقات دانشجويى دانشكاه علوم يزشكى اروميه و ور معاونت

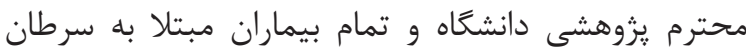

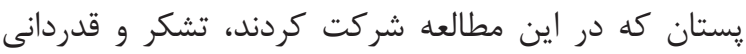
مى شو د.

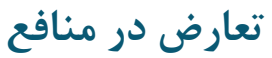

بين نويسندكان در اين مطالعه تضاد منافعى وجود ندارد.

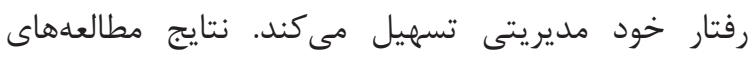

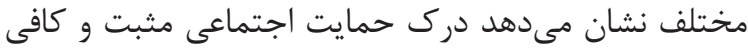

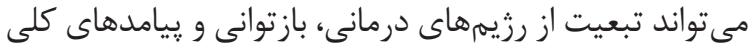

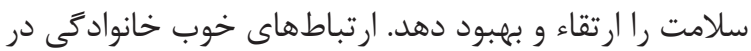

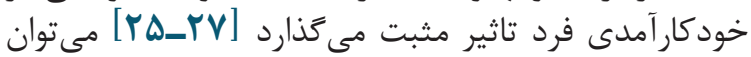

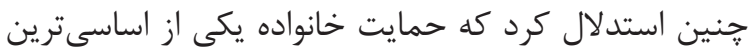

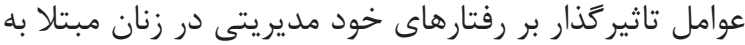
سرطان پستان است.

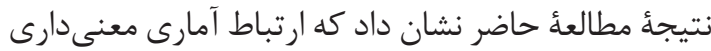

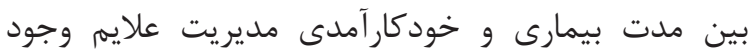

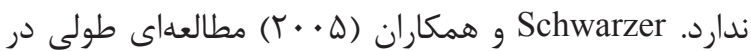

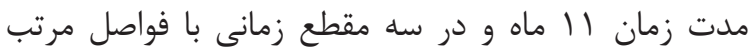

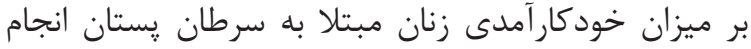

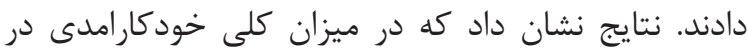

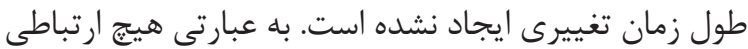

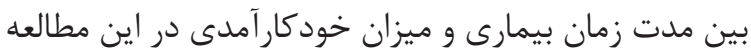

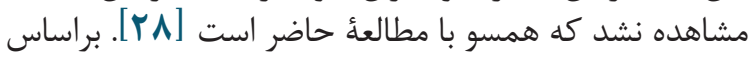

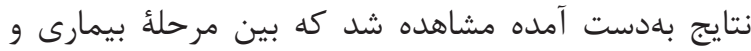

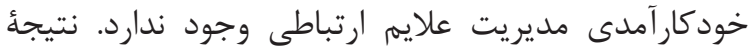

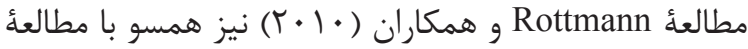

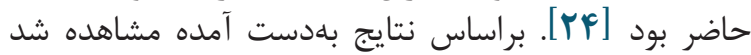

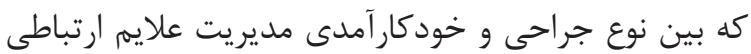

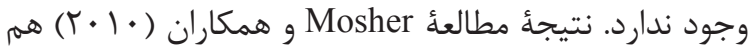

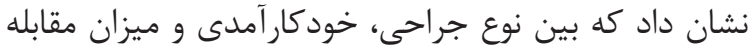

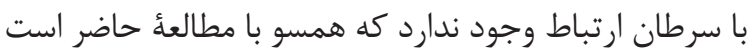

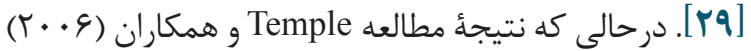

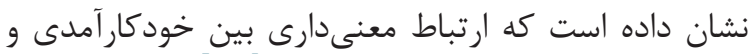

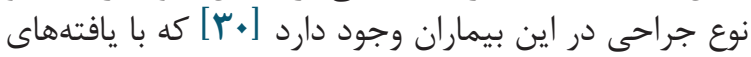

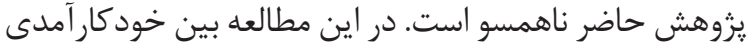

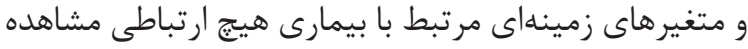

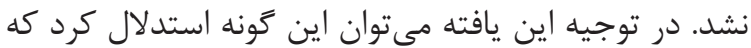

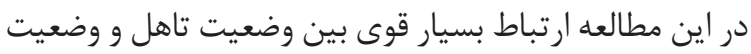

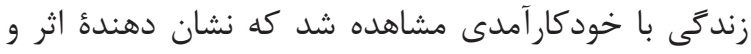

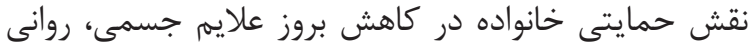

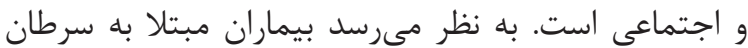

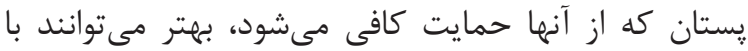

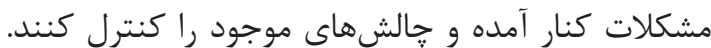

\section{نتيجهل}

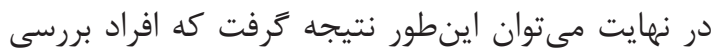

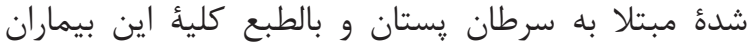

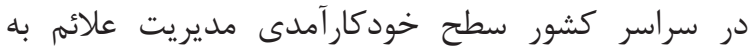

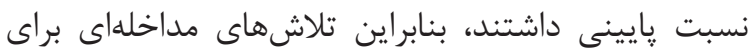

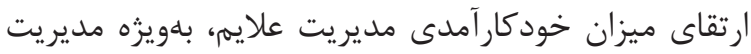

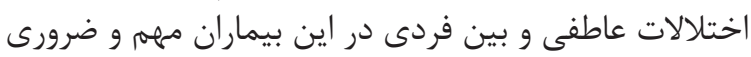

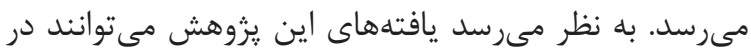

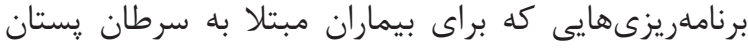

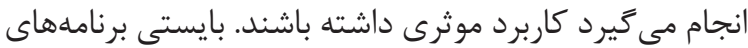

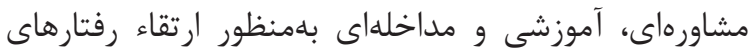




\section{References}

1. DeSantis C, Ma J, Bryan L, Jemal A. Breast cancer statistics, 2013. CA: a cancer journal for clinicians. 2014;64(1):52-62. https://doi. org/10.3322/caac. 21203

2. Feldstain A, Lebel S, Chasen M. An interdisciplinary palliative rehabilitation intervention bolstering general self-efficacy to attenuate symptoms of depression in patients living with advanced cancer. Support Care Cancer. 2015;24(1):10917. https://doi.org/10.1007/s00520-015-2751-4 PMid:25953381

3. Ganz PA, Greendale GA, Petersen L, Zibecchi L, Kahn B, Belin TR. Managing menopausal symptoms in breast cancer survivors: results of a randomized controlled trial. J Natl Cancer Inst. 2000;92(13):1054-64. $\quad$ https://doi.org/10.1093/ jnci/92.13.1054 PMid:10880548

4. Shayan A, Khalili A, Rahnavardi M, Masoumi SZ. The relationship between sexual function and mental health of women with breast cancer. Sci J Hamadan Nurs Midwifery Fac. 2016;24(4):2218. https://doi.org/10.21859/nmj-24042

5. Tighe M, Molassiotis A, Morris J, Richardson J. Coping, meaning and symptom experience: A narrative approach to the overwhelming impacts of breast cancer in the first year following diagnosis. Eur J Oncol Nurs. 2011;15(3):22632. https://doi.org/10.1016/j.ejon.2011.03.004 PMid: 21511530

6. Partridge AH, Burstein HJ, Winer EP. Side effects of chemotherapy and combined chemohormonal therapy in women with early-stage breast cancer. J Natl Cancer Inst Monogr. 2001;(30):13542. https://doi.org/10.1093/oxfordjournals.jncimonographs.a003451 PMid: 11773307

7. Lam WWT, Fielding R. Is self-efficacy a predictor of short-term post-surgical adjustment among Chinese women with breast cancer. Psychooncology. 2007;16(7):651-9.

8. Bandura A. Self-efficacy: Wiley Online Library; 1994. PMid: 8147544

9. Philip EJ, Merluzzi TV, Zhang Z, Heitzmann CA. Depression and cancer survivorship: importance of coping self-efficacy in post-treatment survivors. Psychooncology. 2013;22(5):987-94. https://doi. org/10.1002/pon.3088 PMid:22573371 PMCid:PMC3432138

10. Akin S, Can G, Durna Z, Aydiner A. The quality of life and self-efficacy of Turkish breast cancer patients undergoing chemotherapy. Eur J Oncol Nurs. 2008;12(5):449-56. https://doi. org/10.1016/j.ejon.2008.07.006 PMid: 18842460

11. Hirai K, Suzuki Y, Tsuneto S, Ikenaga M, Hosaka T, Kashiwagi T. A structural model of the relationships among self-efficacy, psychological adjustment, and physical condition in Japanese advanced cancer patients. Psychooncology.2002;11(3):2219.https://doi.org/10.1002/pon.561 PMid: 12112482

12. Hosseini S S DJ, Habibi M, Philding R. Comparison of the expected outcome of the mastectomy with perceived treatment outcomes based on self-efficacy in women with breast cancer. Health Psychology. 2014;3(12):70-83.

13. Kazemi M MJm. Comparative study of the efficacy and the empowerment of women with breast cancer and healthy. Iranian Journal of Breast Disease. 2012;5(1).

14. Rasooli ES, Rahmani A, Zamanzadeh V, Ashrafi R, Nikfar AR, Heidarzadeh H. Relationship self-efficacy with perception of prognosis among cancer patients. Iranian Journal of Psychiatric Nursing. 2014;2(2):33-45.

15. Shelby RA, Edmond SN, Wren AA, Keefe FJ, Peppercorn JM, Marcom PK, et al. Self-efficacy for coping with symptoms moderates the relationship between physical symptoms and well-being in breast cancer survivors taking adjuvant endocrine therapy. Support Care Cancer. 2014;22(10):2851-9. https://doi.org/10.1007/ s00520-014-2269-1 PMid:24821365

16. Liang S-Y, Wu W-W, Kuo C-Y, Lu Y-Y. Development and Preliminary Evaluation of Psychometric Properties of Symptom-Management Self-Efficacy Scale for Breast Cancer Related to Chemotherapy. Asian Nurs Res (Korean Soc Nurs Sci). 2015;9(4):312-7. https://doi.org/10.1016/j. anr.2015.09.001 PMid:26724240

17. Champion VL, Ziner KW, Monahan PO, Stump TE, Cella D, Smith LG, et al., editors. Development and psychometric testing of a breast cancer survivor self-efficacy scale. Oncol Nurs Forum. 2013;40(6):E403-10. https://doi.org/10.1188/13. ONF.E403-E410 PMid:24161644 PMCid:PMC4869969

18. Kiaei MZ, Ferdosi M, Moradi R, Chelongar Q, Ahmadzadeh MS, Bahmanziari N. Association between self-efficacy and quality of life in women with breast cancer undergoing chemotherapy. J Qazvin Univ Med Sci. 2016;20(2):58-65.

19. Paxton RJ, Gao Y, Herrmann SD, Norman GJ. Measurement Properties of the Sedentary Behavior Strategy Self-management Instrument in African-American Breast Cancer Survivors. Am J Health Behav. 2015;39(2):175-82. https://doi. org/10.5993/AJHB.39.2.3 PMid:25564829

20. Tabrizi FM. Health promoting behavior and influencing factors in Iranian breast cancer survivors. Asian Pac J Cancer Prev. 2015;16(5):1729-36. https://doi.org/10.7314/APJCP.2015.16.5.1729 PMid:25773817

21. Ziner KW, Sledge Jr GW, Bell CJ, Johns S, 
Miller KD, Champion VL, editors. Predicting fear of breast cancer recurrence and self-efficacy in survivors by age at diagnosis. Oncology nursing forum. 2012;39(3):287-95. https://doi. org/10.1188/12.ONF.287-295 PMid:22543387 PMCid:PMC5018900

22. Brunault P, Champagne AL, Huguet G, Suzanne I, Senon JL, Body G, et al. Major depressive disorder, personality disorders, and coping strategies are independent risk factors for lower quality of life in non-metastatic breast cancer patients. Psychooncology. 2015;25(5):513-20. https://doi. org/10.1002/pon.3947 PMid:26356037

23. Tamagawa R, Giese-Davis J, Speca M, Doll R, Stephen J, Carlson LE. Trait Mindfulness, Repression, Suppression, and Self-Reported Mood and Stress Symptoms Among Women With Breast Cancer. J Clin Psychol. 2013;69(3):26477.https://doi.org/10.1002/jclp.21939 PMid:23280695

24. Rottmann N, Dalton SO, Christensen J, Frederiksen K, Johansen C. Self-efficacy, adjustment style and well-being in breast cancer patients: a longitudinal study. Qual Life Res. 2010;19(6):82736. https://doi.org/10.1007/s11136-010-9653-1 PMid:20401551

25. Given BA, Given CW, Kozachik S. Family support in advanced cancer. CA Cancer J Clin. 2001;51(4):213-31. https://doi.org/10.3322/canjclin.51.4.213 PMid: 11577488

26. Forsythe LP, Alfano CM, Kent EE, Weaver
KE, Bellizzi K, Arora N, et al. Social support, self-efficacy for decision-making, and follow-up care use in long-term cancer survivors. Psychooncology. 2014;23(7):788-96. $\quad$ https://doi. org/10.1002/pon.3480 PMid:24481884 PMCid:PMC4082440

27. Moradi N, Abdollahzadeh F, Rahmani A, Zamanzadeh V, Asvadi I, Ghaleban K. Effects of husband's education on meting supportive care needs of breast cancer patients: A clinical trial. Sci J Hamadan Nurs Midwifery Fac. 2013;21(3):4050 .

28. Schwarzer R, Boehmer S, Luszczynska A, Mohamed NE, Knoll N. Dispositional self-efficacy as a personal resource factor in coping after surgery. Pers Individ Dif. 2005;39(4):807-18. https://doi.org/10.1016/j.paid.2004.12.016

29. Mosher CE, DuHamel KN, Egert J, Smith MY. Self-efficacy for coping with cancer in a multiethnic sample of breast cancer patients: associations with barriers to pain management and distress. Clin J Pain. 2010;26(3):227-34. https://doi.org/10.1097/AJP.0b013e3181bed0e3 PMid:20173437 PMCid:PMC2827812

30. Temple WJ, Russell ML, Parsons LL, Huber SM, Jones CA, Bankes J, et al. Conservation surgery for breast cancer as the preferred choice: a prospective analysis. J Clin Oncol. 2006;24(21):3367-73. https://doi.org/10.1200/ $\underline{\text { JCO.2005.02.7771 PMid: } 16849750}$ 